\title{
The Cost Effectiveness of Stockpiling Drugs, Vaccines and Other Health Resources for Pandemic Preparedness
}

\author{
Pedro Plans-Rubió ${ }^{1}$ (D) \\ Published online: 4 July 2020 \\ (c) The Author(s) 2020
}

The emergence of a novel coronavirus, designated as severe acute respiratory syndrome coronavirus 2 (SARS-CoV-2), in Wuhan (China) at the end of 2019 is a worldwide public health challenge [1]. Several pandemic and potentially pandemic pathogens have emerged in the last 30 years, including coronaviruses SARS-CoV and MERS-CoV, influenza viruses H1N1 and H7N9 and Ebola viruses.

The emergence of the novel COVID-19 pandemic raises two questions: What are available measures or resources for pandemic preparedness? What is the best pandemic preparedness strategy to prevent and mitigate pandemic effects from a cost-effectiveness point of view? Pandemic preparedness strategies include four types of measures: (1) measures to detect infected individuals, (2) measures to treat patients, (3) measures to prevent infections and (4) measures to prevent transmission of pandemic pathogens. Measures to detect infected patients include laboratory detection tests, from enzyme-linked immunosorbent assays (ELISA) to polymerase chain reaction tests (PCR). Measures to treat patients include hospital beds, intensive care unit (ICU) beds, drugs for treating infected patients and ventilators for respiratory diseases. Measures for preventing infections include vaccines and prophylactic drugs. Measures for preventing transmission of pandemic pathogens include personal protection equipment (PPE) and masks.

Pandemic preparedness plans must indicate required national stockpiles of measures against future pandemics, including drugs, vaccines, prevention equipment and other health resources. In the United States, the National Pandemic Plan [2] considered it necessary to allocate resources to stockpile 81 million treatment courses of approved antiviral drugs and 40 million doses of vaccines against influenza virus subtypes considered to pose a substantial pandemic

Pedro Plans-Rubió

pedro.plans@gencat.cat

1 Department of Health of Catalonia, Public Health Agency of Catalonia, Roc Boronat 83-95, 08005 Barcelona, Spain risk (currently avian H5N1). In Europe, many countries have developed pandemic preparedness plans. An evaluation of pandemic preparedness plans in European countries carried out by the WHO's Regional Office for Europe [3], after the influenza pandemic of 2009, found the following weaknesses: poor seasonal influenza vaccination coverage, inequitable access to vaccines across Europe, lack of capacity in intensive care and paediatric services, poor use of antivirals to treat influenza patients and lack of effectiveness of new interventions introduced during the pandemic. These weaknesses are explained by insufficient investments to increase the capacity in intensive care services, insufficient stockpiling of antiviral drugs, insufficient production and distribution of influenza vaccines and insufficient research on treatments against potential new pathogens.

The COVID-19 pandemic has caused a shortage of hospital beds, ICU beds, antiviral drugs, PPE, masks, ventilators and other health resources in many countries [4]. Why did pandemic preparedness measures receive insufficient investments? The answer is that investments in pandemic preparedness plans, including different types of measures, must be done now and their return will come in the future. For this reason, it is necessary to develop consistent evaluative studies comparing costs and effects of different pandemic preparedness measures, including stockpiling of drugs against potential pandemic pathogens, stockpiling of personal protection equipment (PPE) and masks and stockpiling of ventilators for treating respiratory diseases.

Health economics methods can be used to decide the optimal pandemic preparedness strategy based on cost effectiveness because different stockpiling of available measures can be implemented. The economic evaluation of pandemic preparedness strategies and pandemic preparedness measures is based on methods developed for health technology assessment. Nevertheless, this assessment differs from the traditional economic evaluations. The cost-effectiveness evaluation of a new drug compares healthcare costs and health effects for patients treated and not treated with the 
drug. The cost effectiveness of the drug will depend on the effectiveness of the drug in reducing clinical outcomes and healthcare costs. The drug will be used by the health system in patients with certainty. In contrast to this, the costeffectiveness evaluation of pandemic preparedness measures and interventions is affected by several facts. First, pandemic preparedness measures are costly because they must be used to prevent and treat pandemic infections in a great number of persons. Second, investments in pandemic preparedness measures could be made many years before the emergence of the pandemic pathogen. Third, the health and economic benefits generated by pandemic preparedness measures will depend on the virulence and infectiousness of the pandemic pathogen. Fourth, pandemic preparedness measures can be associated with large costs and benefits outside the health system and great macroeconomics effects. There is a risk that an unknown pandemic agent will emerge and cause high morbidity and mortality, but we do not know when this will happen or how virulent and infectious a new pandemic agent will be. Although a new pandemic can be similar to previous pandemics, it can be also very different.

Several evaluative studies have found that stockpiling of antiviral drugs against influenza is a cost-effective intervention. Milne et al. [5] assessed the cost effectiveness in terms of cost per life-year saved (LYS) of interventions against influenza pandemics including school closure, antiviral drugs for treatment and prophylaxis, workplace non-attendance and community contact reduction in the United States in 2012. Three antiviral drug strategies were examined: (1) antiviral treatment of influenza cases, (2) antiviral treatment of influenza cases and antiviral prophylaxis of household contacts of influenza cases and (3) antiviral treatment of influenza cases, antiviral prophylaxis of household contacts of influenza cases and antiviral prophylaxis of school or workplace contacts of influenza cases. The study assumed that influenza pandemics were associated with clinical attack rates (percentage of the population contracting the clinical disease) of $32 \%$ and infection transmissibility in terms of secondary cases per infected case (basic reproduction number, Ro) of 1.8. The most cost-effective intervention against severe influenza pandemics $(0.75-2.5 \%$ case fatality rate) included continuous school closure, antiviral treatment of symptomatic patients, prophylaxis of household contacts of influenza cases and 50\% community contact reduction. The cost effectiveness of this intervention was US $\$ 8550$ per LYS in very high severity pandemics and US $\$ 13,447$ per LYS in moderate severity pandemics. The most cost-effective intervention against low-severity influenza pandemics $(0.03-0.25 \%$ case fatality rate) included the same combined intervention plus antiviral prophylaxis of school or workplace contacts of influenza cases. The costeffectiveness ratio for this intervention was US\$26,726 per LYS in low-severity pandemics and US\$154,908 per LYS in very-low-severity pandemics. The sensitivity analyses found that the results of the study hold for variations in key parameters. Varying the reproduction number from 1.8 to 1.5 and from 1.8 to 2.5 resulted in higher and lower costeffectiveness ratios for assessed interventions, respectively, but it did not change the relative cost effectiveness of the assessed intervention.

Siddiqui and Edmunds [6] assessed the cost effectiveness of stockpiling the neuraminidase inhibitor oseltamivir for a potential influenza pandemic in the UK in 2004. The study selected oseltamivir because it was cheaper and easier to stockpile than zanamivir, an alternative neuraminidase inhibitor, and it dominated zanimavir in cost-utility analysis. Cyclic amines (amantadine and rimantadine) were not included in the analysis because influenza viruses can be resistant to cyclic amines. The study assessed the cost effectiveness of three pandemic strategies: (1) do not treat with antiviral drugs (no intervention), (2) treat all influenza-like patients with antiviral drugs (treat only intervention) and (3) test all influenza-like patients for influenza and treat only individuals with a positive test result (test-treat intervention). The study assumed $25 \%$ for the clinical attack rate over one influenza wave lasting 15 weeks, case fatality rates of 0.3\% (1957/69 pandemic) and 2.3\% (1918 pandemic), 70\% of influenza-like patients received antiviral drugs within $48 \mathrm{~h}$ of symptomatic onset, and shelf-life of 5 years for antiviral drugs. The test-treatment strategy assumed values of $89.5 \%$ sensitivity and $99.8 \%$ specificity and 2 years' shelf life. The study found that stockpiling 14.6 million courses over 30 years (to treat $87 \%$ of influenza-like patients) was a cost-effective pandemic preparedness intervention because it was associated with an incremental cost-effectiveness ratio (compared with do not treat) $<£ 30,000$ per QALY. The test-treat strategy was not a cost-effective intervention because it was associated with an incremental cost-effective ratio (compared with treat only) $>£ 30,000$ per QALY. The univariate sensitivity analysis showed that the cost effectiveness of stockpiling antiviral drugs depended on the timing of the pandemic and the pandemic case death rate. The incremental cost-effectiveness ratio increased when the timing of the pandemic was in $<30$ years and decreased when it was in $>30$ years, but stockpiling antiviral drugs was also a cost-effective intervention when the pandemic occurred in 45 years: $£ 3800$ per QALY and $£ 28,000$ per QALY for the 1918 and 1957/69 pandemic scenarios, respectively. Stockpiling antiviral drugs would remain cost effective if the probability of receiving antiviral drugs within $48 \mathrm{~h}$ was not $<35 \%$. Varying the clinical attack rate from 28 to $10-40 \%$ did not significantly change the cost effectiveness of stockpiling antiviral drugs. The probability sensitivity analysis, where all model parameters were varied, showed that stockpiling antiviral drugs was a cost-effective pandemic 
preparedness intervention with a high probability, irrespective of the case fatality scenario.

In pandemics due to air-transmitted infections, such as influenza and coronavirus, ventilators in intensive care units are of critical necessity to reduce mortality in infected patients. Ventilators are costly but can reduce deaths from COVID-19 infections [2, 3]. In the United States, in 2010, there were 62,000 full-featured ventilators in hospitals; 10,000-20,000 were available from the Strategic National Stockpile, a federal cache of supplies and medicines held in case of emergencies; and 98,000 not fully featured ventilators could be used during a crisis [7]. However, the National Pandemic Plan of the United States considered that a severe influenza pandemic would require mechanical ventilators for 740,000 critically ill people [2]. Ventilator support and intensive care for acute respiratory failure due to acute respiratory distress syndrome is a cost-effective intervention [8], but the cost effectiveness of stockpiling ventilators depends on the number of stockpiled ventilators and the severity of a future pandemic. The cost effectiveness of ventilator support and intensive care ranges from US $\$ 29,000$ per QALY in low-risk patients ( $\geq 70 \%$ probability of surviving at least 2 months from the time of ventilator support) to US\$110,000 per QALY in high-risk patients (prognostic estimate $\leq 50 \%$ ) [7]. The question about the optimal number of stockpiling ventilators for pandemic preparedness depends on intervention costs and uncertainty about when the pandemic will happen and how virulent and infectious the pandemic pathogen will be.

Evaluative studies assessing the cost effectiveness of pandemic preparedness measures and strategies are necessary to demonstrate their effectiveness and efficiency in reducing pandemic effects.

\section{Compliance with Ethical Standards}

Funding The paper was not funded.

Conflict of interest I declare that I have received a lecture fee from Seqirus.
Open Access This article is licensed under a Creative Commons Attribution-NonCommercial 4.0 International License, which permits any non-commercial use, sharing, adaptation, distribution and reproduction in any medium or format, as long as you give appropriate credit to the original author(s) and the source, provide a link to the Creative Commons licence, and indicate if changes were made. The images or other third party material in this article are included in the article's Creative Commons licence, unless indicated otherwise in a credit line to the material. If material is not included in the article's Creative Commons licence and your intended use is not permitted by statutory regulation or exceeds the permitted use, you will need to obtain permission directly from the copyright holder. To view a copy of this licence, visit http://creativecommons.org/licenses/by-nc/4.0/.

\section{References}

1. Zhu N, Zhang D, Wang W, Li X, Yang B, Song J, et al. A Novel Coronavirus from patients with pneumonia in China, 2019. N Engl J Med. 2020;382:727-33. https://doi.org/10.1056/NEJMo a2001017.

2. US Department of Health and Human Services. HHS Pandemic Influenza Plan. Washington, DC: US Department of Health and Human Services, 2005. https://www.cdc.gov/flu/pdf/profession als/hhspandemicinfluenzaplan.pdf. Accessed 5 June 2020.

3. Nicoll A, Brown C, Karcher F, Pentinen P, Hegermann-Lindencrone $\mathrm{M}$, Villanueva $\mathrm{S}$, et al. Developing pandemic preparedness in Europe in the 21st century: experience, evolution and next steps. Bull World Health Organ. 2012;90:311-7.

4. Wikipedia. Shortages related to the COVID-19 pandemic. https ://en.wikipedia.org/wiki/Shortages_related_to_the_COVID-19_ pandemic. Accessed 8 June 2020.

5. Milne GJ, Halder N, Kelso JK. The cost effectiveness of pandemic influenzainterventions: a Pandemic severity based analysis. PLoS ONE. 2013;8(4):e61504. https://doi.org/10.1371/journ al.pone.0061504.

6. Siddiqui MR, Edmunds WJ. Cost-effectiveness of antiviral stockpiling and near-patient testing for potential influenza pandemic. Emerg Infect Dis. 2008;14:267-74.

7. Rubinson L, Vaughn F, Nelson S, et al. Mechanical ventilators in US acute care hospitals. Disaster Med Public Health Prep. 2010;4:199-206.

8. Hamel MB, Phillips RS, Davis RB, Teno J, Connors AF, Desbiens $\mathrm{N}$, et al. Outcomes and cost-effectiveness of ventilator support and aggressive care for patients with acute respiratory failure due to pneumonia or acute respiratory distress syndrome. Am J Med. 2000;109:614-20. https://doi.org/10.1016/s0002-9343(00)00591 $-\mathrm{x}$. 